\section{Weniger Brustkrebs unter ASS-Therapie}

Kalifornische Forscher untersuchten anhand von prospektiv erhobenen Daten von rund 57.000 Frauen den Zusammenhang zwischen einer regelmäßigen ASSEinnahme und Brustkrebs. 23\% aller Frauen schluckten mindestens dreimal in der Woche Niedrig-Dosis-ASS (81 mg/d). Ihr Brustkrebsrisiko war, unter Berücksichtigung bekannter Risikofaktoren, um 16\% geringer als das von Frauen ohne NSARTherapie. Dafür maßgeblich war ein Rückgang des Risikos für HR-positive/HER2-negative Tumoren um 20\%. Bei Tumoren mit anderem Rezeptorstatus war kein Vorteil der ASS-Therapie zu erkennen.

Clarke CA et al. Breast Cancer Res. 2017;19:52

\section{Übergewicht fördert Risiko für Pankreas-Ca.}

Anhand einer Gendatenbank mit über 7000 Pankreaskarzinompatienten und ebenso vielen Kontrollpersonen ohne solche Tumoren berechneten französiche Forscher, dass jede Erhöhung des BMI um eine Standardabweichung $(4,6$ $\mathrm{kg} / \mathrm{m}^{2}$ ) das Risiko für ein Pankreas-Ca. um ein Drittel (34\%) erhöht. Außerdem berechneten sie pro zusätzliche Standardabweichung $(44,4 \mathrm{pmol} / \mathrm{l})$ der Nüchterninsulinwerte eine Erhöhung des Tumorrisikos um 66\%. Dies betraf jedoch nur Männer.

Carreras-Torres Ret al. J Natl Cancer Inst 2017;09(9)

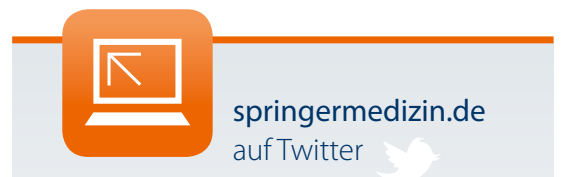

Auch im Sommer heißt es wieder:

Lauschen Sie unserem Zwitschern und werden Sie zum Follower.

- twitter.com/springermedizin

Aktivität des braunen Fettgewebes im Blick

\title{
Höhere Diabetesinzidenz durch Erderwärmung?
}

Die globale Erwärmung geht niederländischen Berechnungen zufolge mit einem Anstieg der Diabetesinzidenz einher. Die Wissenschaftler vermuten einen Zusammenhang mit der Aktivität des braunen Fettgewebes.

Niederländische Forscher stellten die Hypothese auf, dass die globale Erwärmung zum Anstieg der Inzidenz des Typ-2-Diabetes beiträgt. Sie analysierten Daten zur Diabetesinzidenz aus den Jahren 1996 bis 2009 aus 50 US-Staaten sowie Guam, Puerto Rico und Virgin Islands.

Ihren Berechnungen nach nimmt die Diabetesinzidenz - altersadjustiert - bei einem Temperaturanstieg um $1^{\circ} \mathrm{C}$ um 0,314 (95\%-Konfidenzintervall [KI] 0,194-0,434) pro 1000 Personen zu. Al- lein in den USA treten somit jährlich mehr als 100.000 neue Diabeteserkrankungen auf. Weltweit sei zudem die Prävalenz der Glukoseintoleranz pro $1^{\circ} \mathrm{C}$ um 0,17\% (95\%-KI 0,107\%-0,234\%) gestiegen, schreiben die Wissenschaftler. Diese Zusammenhänge blieben bestehen, wenn bei der Berechnung Daten zur Adipositas berücksichtigt wurden.

Ein kausaler Zusammenhang lässt sich auf Basis dieser Analyse nicht feststellen. Die Forscher spekulieren jedoch, dass es einen Zusammenhang zwischen dem Befund und der Aktivität des durch Kälte aktivierten braunen Fettgewebes geben könnte.

Blauw LL et al. BMJ Open Diabetes Research and Care 2017;5:e000317. doi: 10.1136/bmjdrc-2016-000317

HIV- und HCV-positive Patienten

\section{Keine Panik bei Nadelstichverletzung}

Das Infektionsrisiko nach Kontakt mit Körperflüssigkeiten eines HCV- oder HIV-infizierten Patienten durch Verletzungen von Haut oder Schleimhäuten scheint für das medizinische Personal deutlich geringer zu sein als bislang angenommen.

Mediziner der Universität Pittsburgh, verfolgten über 13 Jahre alle Fälle beruflicher HCV- und HIV-Expositionen an ihrer Klinik. In diesem Zeitraum wurden 1361 Fälle eines HCV- und 266 Fälle eines HIV-Kontakts registriert. Nach den Vorgaben der Klinik erfolgte bei den Verletzten direkt nach der Exposition eine 20-minütige Wunddekontamination. 21,2\% des medizinischen Personals führten nach HIV-Kontakt eine Postexpositionsprophylaxe durch. Die Betroffenen wurden insgesamt sechs Monate alle vier Wochen auf HCV bzw. HIV-Antikörper getestet.

Lediglich zwei der Personen mit HCVKontakt hatten sich tatsächlich mit HCV infiziert, dies entspricht einer Serokonversionsrate von $0,1 \%$. Bei keinem einzigen Verletzten kam es zu einer HIVSerokonversion. Dies sei laut Autoren trotz der gestiegenen Zahl an Menschen, die mit HIV leben, der Einführung der antiretroviralen Therapie zu verdanken, die durch Senkung der Viruslast bei den Patienten auch das Übertragungsrisiko auf das medizinische Personal verringere. st

Egro FM et al. AJIC 2017; online 24 April 2017 DOl: http://dx.doi.org/10.1016/j.ajic.2017.03.011 Nwaiwu CA et al. AJIC 2017; online 24 April 2017 DOl:http://dx.doi.org/10.1016/j.ajic.2017.03.012 